\section{PM0-008 THE VALUE OF ENDOSCOPY IN PATIENTS WITH CONFIRMED DIVERTICULAR DISEASE ON CT SCAN}

doi:10.1136/gutjnl-2012-302514b.8

C Daker, ${ }^{*}$ T Brier, K Besherdas. Department of Gastroenterology, Chase Farm Hospital, London, UK

Introduction Diverticulitis, the common clinical complication of diverticulosis, may affect $10 \%-25 \%$ of patients with colonic diverticula. The diagnosis of diverticulitis may be made on clinical grounds. However, it is usual practice to perform a CT scan to confirm the diagnosis and assess for complications (eg, abscess, fistula, obstruction). CT criteria suggestive of diverticulitis include: presence of diverticula with pericolic infiltration of fatty tissue, thickening of the colonic wall, and abscess formation. The sensitivity of CT scan in diagnosing of diverticulitis is up to $97 \%$. Once an episode of diverticulitis has been treated, we have observed patients to be followed up by undergoing routine colonoscopy (CSy). We hypothesised that the value of CSy in patients with a confirmed CT diagnosis of diverticulitis is negligible.

Methods A duel centre (2 North London hospitals), retrospective analysis of all patients with an ICD 10 coding on their in-patient discharge summary letters of diverticular disease (DD) over the past year was employed. Patient notes were scrutinised and correlated to endoscopic records. The dates of both CT scan and CSy were recorded. Completion rates of the endoscopies and findings in addition to diverticular disease was noted.

Results 137 patients over a 4-month period had DD recorded within the discharge summary. 47 patients with presumed diagnosis of diverticulitis had a CT scan prior to endoscopy (35 CSy and/12 Flexible sigmoidoscopy). Of the 47, DD was evident on the CT scan in 34. Endoscopy subsequently confirmed DD in $32 / 34$ of these cases (95\%), with four procedures ending in failure. No other pathological features were found in 30/34 cases. Within the cohort of 32 patients in which endoscopy confirmed CT, three had colonic polyps. Of the 13 cases (of 47) in which DD was not reported on the prior CT scan, subsequent endoscopy confirmed DD in $11 / 13$, with one procedure ending in failure. Of these 11 cases, one had a colonic polyp. In the two cases where endoscopy did not reveal DD, a diagnosis of colitis was recorded.

Conclusion In this study, performing a CSy in patients previously diagnosed with diverticulitis confirmed on CT scan add no further information. CSy is only useful in the setting of clinical diverticulitis if the diagnosis is not supported by CT scan. In patients with diverticulitis other diagnosis such as polyps were detected in only 4 of 47 patients (8\%), not a unsurprising finding as one would expect to find polyps at routine CSy in upto $25 \%$. From this study, we would not support performing a CSy in patients with clinical diverticulitis confirmed on CT scan and avoiding CSy could save 100 colonoscopies per annum in a hospital like ours freeing up space to perform other procedures with more appropriate indications.

Competing interests None declared.

\section{PM0-009 ARE DIETITIANS' RECOMMENDATIONS FOR THE POST-DISCHARGE SUPPORT OF MALNOURISHED PATIENTS CARRIED OUT IN THE COMMUNITY?}

doi:10.1136/gutjnl-2012-302514b.9

${ }^{1} \mathrm{D}$ Coleman, ${ }^{1} \mathrm{C}$ Baldwin, ${ }^{2} \mathrm{C}$ E Weekes. ${ }^{* 1}$ Department of Nutrition \& Dietetics, King's College London, London, UK; ${ }^{2}$ Department of Nutrition \& Dietetics, Guy's \& St Thomas' NHS Foundation Trust, London, UK

Introduction Disease-related malnutrition is common and is often identified during a hospital admission. Hospital stays tend to be short and therefore it is crucial that nutritional interventions begun in hospital are continued on discharge (Elia et al 2010). Research has highlighted failings in nutritional care across this boundary (van Bokhorst-de van der Schueren et al 2005; Bavelaar et al 2008). The aim of this study was to evaluate the continuation of dietetic interventions across the transition from acute to community care. Methods All patients admitted to the acute medical wards and referred to a dietitian for nutrition support between 1 July and 30 September 2011 were considered eligible for this study. Patients were excluded if they died within 1-month of discharge, received enteral or parenteral nutrition, were receiving dietetic care for a long-term chronic condition or were still in hospital at 31 October 2011. Eligible patients or their carers were contacted to determine whether recommendations for their post-discharge oral nutritional support had been carried out. Data were analysed using SPSS V.17.0

Results Of 108 patients, 27 (25\%) died before contact could be made and $17(16 \%)$ did not meet the inclusion criteria. 64 patients were included in this study of whom $35(56 \%)$ were recommended one or more post-discharge dietetic interventions, including consumption of oral nutritional supplements and follow-up dietetic appointments. Of the 35 patients, it was not possible to contact 14 (40\%) within the time limits of the study. Contact was made with 21 patients of whom 17 (81\%) received all the interventions recommended by the dietitian. Of the four patients who did not receive the recommended interventions, in $3(75 \%)$ this was due to patient perception that treatment was no longer required. Of the 64 patients who met the inclusion criteria no comments were included in the discharge letter from the medical team on either nutritional status or dietetic input.

Conclusion In this study it was possible to contact only a small sample of eligible patients however, of those who were contacted the majority had received the post-discharge interventions recommended by the dietitian. Further studies are required to determine if dietetic recommendations are as likely to be carried out in patients who are more difficult to contact post discharge.

Competing interests None declared.

\section{REFERENCES}

1. Bavelaar JW, Otter CD, van Bodegraven AA, et al. Clin Nutr 2008;27:431-8.

2. Elia M, Russell CA, Stratton RJ. Proc Nutr Soc 2010;69:470-6.

3. van Bokhorst-de van der Schueren MAE, Klinkenberg M, Thijs A. Eur J Clin Nutr 2005; 59:1129-35.

\section{PM0-010 IMPROVING THE ADVERSE EVENT REPORTING PROCESS FOR BOWEL SCREENING WALES}

doi:10.1136/gutjnl-2012-302514b.10

C Lewis.* Bowel Screening Wales, Public Health Wales, Llantrisant, UK

Introduction Risk management is an essential part of the clinical governance framework and central to this is adverse event reporting. The aim of this study is to increase the number of adverse events reported to Bowel Screening Wales (BSW).

Methods The primary research method included a literature review. The focus for the study was adverse event reporting and recording methods. Referral patterns were recorded prior and post intervention and the data compared. The literature review informed the various processes adopted in an attempt to increase referrals for adverse events from the Local Assessment Centres (LACs). The interventions took place in all LACs throughout February 2011 They included defining and providing examples of adverse events, educating the Specialist Screening Practitioners (SSPs) and providing a more flexible approach for reporting. A file using Microsoft Office Excel was developed to categorise referrals by subject and consequence. Referrals from the beginning of the programme were 